\title{
Zum 10-jährigen Bestehen der Zeitschrift für Außen- und Sicherheitspolitik (ZfAS)
}

\author{
Thomas Jäger • Maja Henke
}

(C) Springer Fachmedien Wiesbaden GmbH, ein Teil von Springer Nature 2019

Mit dieser Ausgabe schließt sich der Kreis der ersten zehn Jahre für unsere Zeitschrift für Außen- und Sicherheitspolitik. Im Herbst 2008 erschien sie erstmals und seitdem gab sie Quartal für Quartal wichtige Diskussionen, unterschiedliche Perspektiven und innovative Ansätze wieder. Wir wollten diese im Austausch zwischen der außen- und sicherheitspolitischen Praxis und den Forschungen an den Thinktanks und Universitäten anlegen und möchten uns ganz herzlich bei allen Autorinnen und Autoren bedanken, die in den letzten zehn Jahren dazu beigetragen haben, dass dies gelungen ist. Eine Zeitschrift lebt von denjenigen, die schreiben, und denjenigen, die lesen. Wir haben uns über zahlreiche Manuskripte gefreut, waren dankbar, wenn von uns angestoßene Themen aufgegriffen wurden und konnten Jahr für Jahr sehen, wie die Resonanz in der Leserschaft zunimmt.

Zwischen Schreiben und Lesen gibt es aber auch noch einen weiteren Prozess - den der redaktionellen Arbeit, der kleine Maschinenraum dieser Zeitschrift, der alles am Leben hält. Das große Engagement und die beruhigende Professionalität aller Mitarbeiterinnen und Mitarbeiter in Redaktion und Redaktionsassistenz haben in den letzten zehn Jahren dafür gesorgt, dass hochwertige Beiträge in einem professionellen Redaktionsprozess verarbeitet werden konnten. Herzlichen Dank dafür!

Ein großes Dankeschön gilt allen Gutachterinnen und Gutachtern, die unsere Qualitätsstandards hochgehalten haben. Ebensolcher Dank geht an die Mitarbeiterinnen und Mitarbeiter bei Springer VS, die sich für unsere ZfAS eingesetzt und die Herstellung mit großer Unterstützung begleitet haben.

Prof. Dr. T. Jäger $\cdot$ M. Henke $(\bowtie)$

Forschungsinstitut für Politische Wissenschaft und Europäische Fragen, Universität zu Köln,

Gottfried-Keller-Str. 6, 50931 Köln, Deutschland

E-Mail: maja.henke@uni-koeln.de

Prof. Dr. T. Jäger

E-Mail: thomas.jaeger@uni-koeln.de 
Vor der Jubiläumsausgabe haben wir den Blick zurück auf zehn Jahre ZfAS geworfen und uns anschließend überlegt, es wäre angemessen und herausfordernd zugleich, verschiedene Staaten und ihre Beziehungen, Konflikte und Politikfelder ebenfalls in diesen Zehn-Jahres-Blick zu nehmen. Wir haben also auf die vergangenen Jahre zurückgeschaut und doch, wie sollte es anders sein, auch gleichzeitig nach vorne. Drei Fragen resultierten hieraus: Wie haben sich grundlegende Themen in den letzten zehn Jahren entwickelt? Wo stehen sie heute? Und wie mögen sie sich in Zukunft gestalten?

Diese drei Fragen gaben wir ausgewiesenen Expertinnen und Experten aus Praxis und Wissenschaft mit an die Hand und baten sie, uns ihre Expertise und ihre Bewertung zu schreiben. Auf diese Weise sollen die Gespräche zur Sache in Gang gesetzt werden. Herausgekommen ist ein wunderbares Heft, das die ursprüngliche Intention der ZfAS, Praxis und Wissenschaft in den Dialog zu bringen und die ganze Bandbreite von außen- und sicherheitspolitischen Themen abzudecken, ausgezeichnet erfüllt. So divers die Themen sind - und nicht alle konnten in einem Heft Platz finden - so unterschiedlich sind unsere Autorinnen und Autoren. Und wer sein Thema in unserem bunten Jubiläumsstrauß vermisst, den laden wir herzlich ein, es aufzugreifen und uns zu schicken. Denn auch das gehört zum Alltag einer Zeitschrift: Während die Jubiläumsausgabe erscheint, sitzen wir bereits an der ersten Ausgabe für die nächste Dekade, in der dann auch ein 10-Jahresrückblick zu Russland erscheinen wird.

Wir freuen uns auf Ihre Beiträge und darauf, dass Sie in unserer ZfAS weiterhin Diskussionen anstoßen und Debatten führen, die uns besser verstehen lassen, was auf den Gebieten der Außen- und Sicherheitspolitik gerade geschieht, wohin sich die Lagen entwickeln und was dies für Menschen, Gesellschaften und Staaten bedeutet.

Prof. Dr. Thomas Jäger

Herausgeber der Zeitschrift für Außen- und Sicherheitspolitik

Maja Henke

Chefredakteurin der Zeitschrift für Außen- und Sicherheitspolitik 